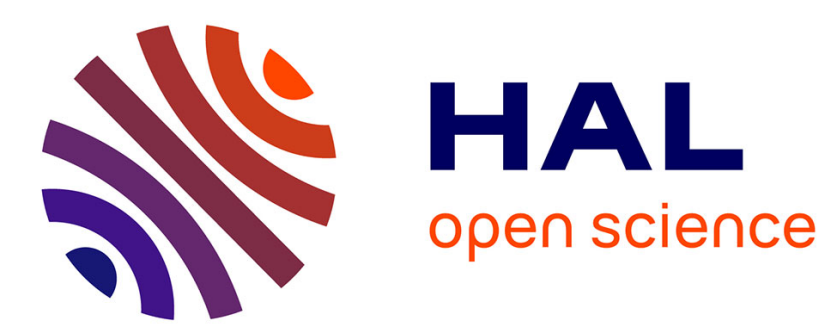

\title{
Stokes Instability in Inhomogeneous Membranes: Application to Lipoprotein Suction of Cholesterol-Enriched Domains
}

\author{
Martine Ben Amar, Jean-Marc Allain, N. Puff, M. Angelova
}

\section{To cite this version:}

Martine Ben Amar, Jean-Marc Allain, N. Puff, M. Angelova. Stokes Instability in Inhomogeneous Membranes: Application to Lipoprotein Suction of Cholesterol-Enriched Domains. Physical Review Letters, 2007, 99 (4), pp.044503. 10.1103/PhysRevLett.99.044503 . hal-02443663

\section{HAL Id: hal-02443663 https://hal.sorbonne-universite.fr/hal-02443663}

Submitted on 9 Mar 2020

HAL is a multi-disciplinary open access archive for the deposit and dissemination of scientific research documents, whether they are published or not. The documents may come from teaching and research institutions in France or abroad, or from public or private research centers.
L'archive ouverte pluridisciplinaire $\mathbf{H A L}$, est destinée au dépôt et à la diffusion de documents scientifiques de niveau recherche, publiés ou non, émanant des établissements d'enseignement et de recherche français ou étrangers, des laboratoires publics ou privés. 


\title{
Stokes Instability in Inhomogeneous Membranes: Application to Lipoprotein Suction of Cholesterol-Enriched Domains
}

\author{
M. Ben Amar, ${ }^{1, *}$ J.-M. Allain, ${ }^{1,2}$ N. Puff, ${ }^{3}$ and M. I. Angelova ${ }^{3}$ \\ ${ }^{1}$ Laboratoire de Physique Statistique, Ecole Normale Supérieure, 24 rue Lhomond, 75231 Paris Cedex 05, France \\ ${ }^{2}$ Laboratoire de Mécanique des Solides, CNRS UMR7649, Département de Mécanique, Ecole Polytechnique, \\ 91128 Palaiseau Cedex, France \\ ${ }^{3}$ Université Pierre et Marie Curie-Paris 6, UMR INSERM 538, CHU Saint-Antoine, 27 rue de Chaligny, F-75012 Paris, France
}

(Received 12 February 2007; published 25 July 2007)

\begin{abstract}
We examine the time-dependent distortion of a nearly circular viscous domain in an infinite viscous sheet when suction occurs. Suction, the driving force of the instability, can occur everywhere in the two phases separated by an interface. The model assumes a two-dimensional Stokes flow; the selection of the wavelength at short times is determined by a variational procedure. Contrary to the viscous fingering instability, undulations of the boundary may be observed for enough pumping, whatever the sign of the viscosity contrast between the two fluids involved. We apply our model to the suction by lipoproteins of cholesterol-enriched domains in giant unilamellar vesicles. Comparison of the number of undulations given by the model and by the experiments gives reasonable values of physical quantities such as the viscosities of the domains.
\end{abstract}

DOI: 10.1103/PhysRevLett.99.044503

Interfacial instabilities have been the subject of numerous experimental and theoretical works in the past [1-3]. They mimic pattern formations, and, in the case of Laplacian growth [4,5], they are well understood. Recently, the sucking instability of a viscous patch in a Hele-Shaw cell has been revisited [6-8], and comparisons with new experiments using a lifting Hele-Shaw cell have been performed [7]. For a two-dimensional Darcy flow, the destabilization of the circular interface $[9,10]$ is easily understood by the Saffman-Taylor instability $[4,11]$, which occurs as soon as a less viscous fluid (usually the air) penetrates a more viscous one (usually the oil). A linear perturbation analysis confirms this interpretation, the agreement of the selected number of oscillations, and the experimental data being only qualitatively correct $[6,8]$. The same suction process for a two-dimensional Stokes flow has received much less attention, probably because of the difficulty to realize a true 2D flow experimentally but also because of the difficulties of the calculations $[12,13]$ when the physical answer is generally expected to be similar to the Laplacian flow. The rapid development of microfluidics [14] and new dynamical experiments involving either Langmuir-Blodgett films [15] or inhomogeneous lipidic membranes [16] prompt us to reexamine the suction instability for a 2D Stokes flow because this model is a good representation of the experimental setups. It turns out that the Stokes instability of suction differs significantly from the Darcy instability [10]. It occurs only for convergent radial flow independently of the sign of the viscosity contrast between the two phases involved. The dispersion relation is also unusual; it is why we perform a selection analysis based on a variational procedure taking into account the balance of capillarity and of viscous stresses to predict the correct number of oscillations. The range of applications of this result is broad, especially as biological
PACS numbers: 47.20.-k, 47.54. $-\mathrm{r}$, 68.03.Cd, 87.16.Dg

processes are involved, and experimental verifications are rather easy. In this Letter, we focus on the suction of an inhomogeneous lipid membrane including a liquid-ordered (LO) phase [17], and we show that such a theory allows us to explain the experiments and to deduce correct values of physical parameters hard to measure for these soft-matter systems.

Theoretical treatment. - We consider a planar biphasic viscous sheet made of a viscous patch of viscosity $\mu_{i}$ ( $i$ for inner) in an infinite two-dimensional bath of viscosity $\mu_{o}$ ( $o$ for outer). The thickness of the sheet $h$ is assumed to be constant; this explains a part of the experimental difficulties. Langmuir-Blodgett films or lipidic membranes whose thickness is chemically determined may correspond to this case, except there is a third phase: the surrounding water bath, of viscosity $\mu_{w}$ (see Fig. 1). Before pumping, the inner domain at rest is circular, with a radius $R_{0}$.

Whatever its origin (chemical or physical), the pumping can occur in both phases. It is described by the extraction rate $q(r)$, which has a radial symmetry around the center of the inner domain. This extraction rate may be timedependent. Once integrated over the whole domain, it gives the total mass extraction per time unit, a quantity easy to measure and control experimentally. The question we ask concerns the existence of a symmetry breaking when the extraction rate increases, inducing a contour fingering not explained by an isotropic pumping process.

Once modified by the water drag [18-20], the Stokes equation becomes in each phase of the membrane:

$$
\begin{aligned}
\vec{\nabla} P_{l} & =\mu_{l} \Delta \vec{V}_{l}-2 \frac{\mu_{w}}{h}\left|\frac{\partial \vec{V}_{w}}{\partial z}\right|_{z=0}, \quad \text { with } \\
\operatorname{div} \vec{V}_{l} & =-q_{l}(r)
\end{aligned}
$$

where $P_{l}$ is the pressure, $V_{l}$ the fluid velocity, $q_{l}(r)$ the 


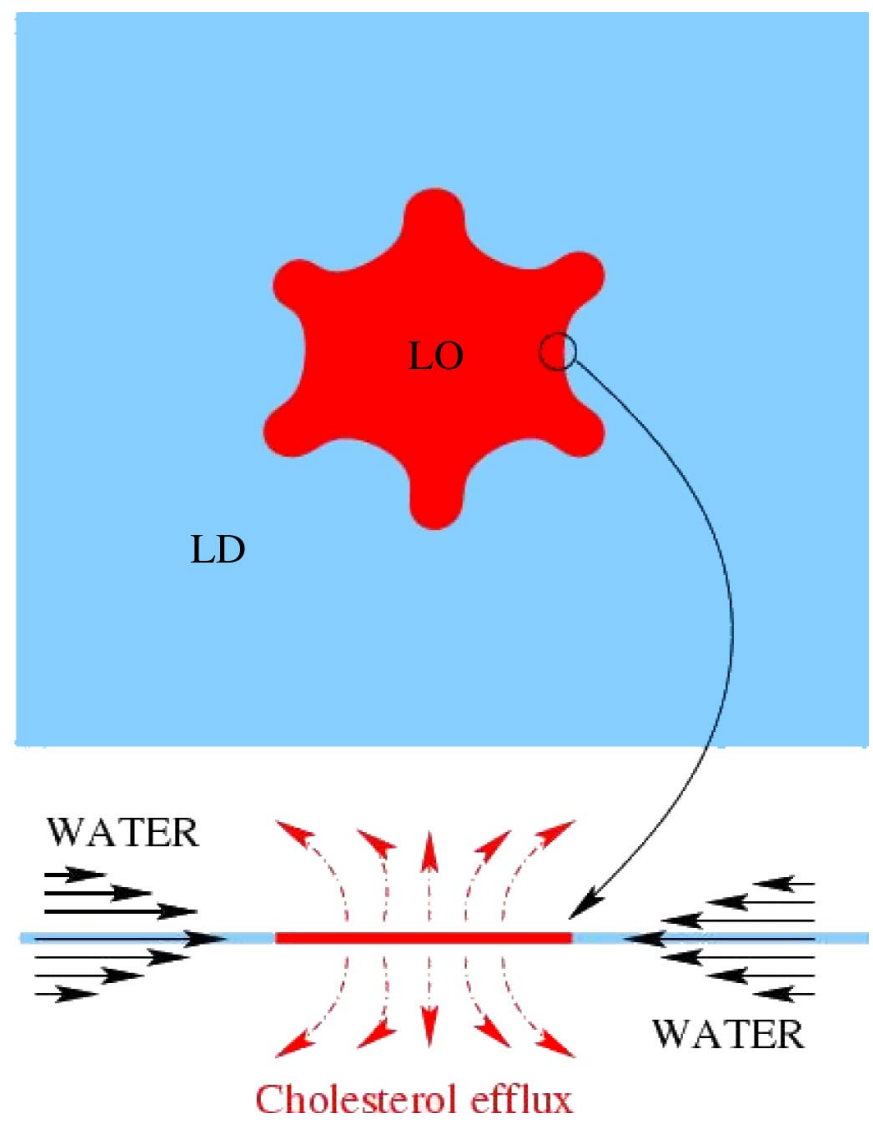

FIG. 1 (color online). Picture of the bidimensional film with a destabilized inner domain, with a side view of the hydrodynamic currents in the three phases involved in the suction process.

local suction rate in the domain $l$, and $V_{w}$ the water velocity induced by the pumping (see Fig. 1). The surrounding water bath is described by the classical 3D Stokes equation for an incompressible fluid. Boundary conditions at the interface are the continuity of the velocity and shear stresses and a jump of normal stress as given by the Laplace law (see [12,13]). If the boundary does not remain circular, we do not have at the moment an analytic solution of the full problem including the water drag, measured by the dimensionless number $N=\left(\mu_{w} R\right) /\left(\mu_{l} h\right)$. But, if in the experiment both phases are sufficiently viscous in such a way that $N$ is small, the water drag can be neglected, and it becomes possible to solve Eq. (1) with the boundary conditions for a circular or nearly circular domain of radius $R(\theta, t)=R_{0}(t)\left[1+\epsilon_{n}^{0} \exp \Omega_{n}(t) \cos n \theta\right]$, with $\epsilon_{n}^{0}$ much smaller than 1 and $n$ an integer, with $n \geq 2$. After a tedious but standard linear perturbation analysis, we find the growth rate $\Omega_{n}$ of the perturbation

$$
\Omega_{n}=\frac{2 Q_{i}}{R_{0}^{2}}-\frac{n}{2} \frac{\gamma}{\left(\mu_{o}+\mu_{i}\right) R_{0}},
$$

where $Q_{i}$ is the suction rate of the inner phase only: $Q_{i}=$ $\int_{0}^{R_{0}} \rho q(\rho) d \rho$. As usual, the surface tension $\gamma$ stabilizes the flow. Instability can occur only if $Q_{i} \geq 0$ (net inward flux) whatever the viscosity difference $\eta=\left(\mu_{i}-\mu_{o}\right) /\left(\mu_{i}+\right.$ $\left.\mu_{o}\right)$, in contrast to the Saffman-Taylor instability $[6,7,11]$. Cholesterol extraction in the liquid-disordered (LD) phase induces also the collapse of the LD phase by chemical transfer from the boundary, but in this case $Q_{i}$ is zero and there is no instability. The relation (2) has been obtained by Cummings, Howison, and King [13] in a more restricted context. However, this dispersion relation is unusual. Remember that, for Darcy flows, we have $\Omega_{n}=$ $\left(2 Q / R_{0}^{2}\right)(1+n \eta / 2)-n\left(n^{2}-1\right) \gamma h_{0}^{2} / 12 R_{0}^{3}\left(\mu_{o}+\mu_{i}\right)$ (see, for example, [6]). To obtain the selected mode, we construct and minimize an energy functional for this suction problem, the associated Euler-Lagrange equations being Eq. (1) with the correct boundary conditions:

$$
\begin{aligned}
\Xi= & \gamma \oint \kappa\left(\vec{V}_{l} \cdot \vec{n}\right) d l+\sum_{l}\left[\Lambda_{l} \iint\left(\operatorname{div} \vec{V}_{l}+q_{l}\right) d S\right. \\
& \left.+\sum_{i j} \iint\left\{-p_{l} \delta_{i j}+\mu_{l}\left(2 e_{i j}-e_{i i} \delta_{i j}\right)\right\} e_{i j} d S\right],
\end{aligned}
$$

with $e_{i j}$ the viscous tensor given by $\frac{1}{2}\left(\partial V_{l, j} / \partial x_{i}+\right.$ $\left.\partial V_{l, i} / \partial x_{j}\right)$ in Cartesian coordinates and $\delta_{i j}$ the Kronecker symbol. Variation of (3) has to be performed with respect to the two velocities in each phase. The curvature $\kappa$ is chosen positive, and $\vec{n}$ is the external normal to the domain boundary. This functional $\Xi$ takes into account the mechanical energy variation per time unit [third integral in Eq. (3)], the capillary energy (first integral), and the loss of mass (second integral) being fixed by the Lagrange multiplier $\Lambda_{l}$. The contribution of mode $n$ is

$$
-\pi \gamma\left[Q / R_{0}\left(2-3 n^{2}\right) / 2+\gamma n\left(n^{2}-1\right) / 2\left(\mu_{i}+\mu_{o}\right)\right]
$$

for the capillary term, while, for the third integral, it gives $\pi\left[\left(\mu_{o}-\mu_{i}\right) Q^{2} / R_{0}^{2}(8 n \eta+6)+\gamma^{2} n\left(n^{2}-1\right) / 2\left(\mu_{i}+\mu_{o}\right)\right]$.

The minimum of $\Xi$ is then obtained for $n_{s}=8 \eta^{2}\left(\mu_{i}+\right.$ $\left.\mu_{o}\right) Q / 3 \gamma R_{0}$, which shows that a contrast of viscosity is necessary for the instability to be observed. In the case where one viscosity is dominant, this selected value is close to the one of the marginal stability mode.

The instability can be observed experimentally only if $n_{s} \geq 2$, which gives a threshold value for $Q_{i}$ which can be tested in experiments. To illustrate our purpose and to test the hydrodynamic viscous properties of lipidic membranes, we choose the cholesterol extraction from a cholesterol-enriched phase in an inhomogeneous giant unilamellar vesicle (GUV). The pumping is achieved by high density lipoproteins (HDLs) [21-23], which in vivo remove the cholesterol from the cell membrane [17].

Heterogeneous GUVs as a model system. - In lipid bilayers or vesicles, lateral cohesion is, in general, weak, and the membrane behaves as a fluid for in-plane displacements, while it is considered as an elastic solid for outof-plane deformations $[16,24]$. Studied for at least 30 years, their elastic properties are now well understood $[25,26]$. 
The hydrodynamics, however, has been much less explored. When vesicles contain cholesterol and sphingomyelin, lipid segregation into domains called rafts in biological cells [27-29] can occur. So inhomogeneous artificial vesicles offer a system with two phases separated by a well-defined boundary. One of the phases, enriched in cholesterol, is called LO. The other, where cholesterol lipids are present at lowest concentration, is less structured and is called LD. Then it is tempting to test the fluid properties of both phases by an interfacial dynamical instability such as mass extraction. The difference with Langmuir-Blodgett monolayers [15,19] comes from the existence of two strongly connected layers surrounded by water for the GUV instead of one, the advantage of the GUV being its obvious biological relevance [27-29].

Experimental setup and results. - To compare the sensitivity of LO and LD phases to HDL-cholesterol pumping [21-23], we add HDLs in the vicinity of inhomogeneous GUVs and observe the decrease up to the collapse of one LO domain [compare Figs. 2(A) and 2(B)]. The vesicles are formed by electroswelling [30] from a mixture of egg yolk phosphatidylcholine (EYPC), egg yolk sphingomyelin (EYSM), and cholesterol (Chol), with a lipophilic membrane probe (Di-Q), with EYPC/Di-Q/EYSM/Chol 55:5:20:20 $\mathrm{mol} / \mathrm{mol}$ as described in Ref. [17]. At room temperature, one or several domains, enriched in Chol and EYSM, are observed since the probe is excluded from them (see Fig. 2). Initially, the LO domains are circular. We added $100 \mu \mathrm{l}$ to $350 \mu \mathrm{l}$ of human HDLs at $2 \mathrm{mg} / \mathrm{ml}$ in apo-A1, the main protein component of HDLs. We always observe a decrease of the LO domain [see Figs. 2(A) and 2(B)], the suction rate increasing with the number of added HDL particles (see Fig. 3). For volumes higher than $350 \mu \mathrm{l}$, a destabilization of the circular shape is observed [see Fig. 2(B) for typical examples of our observations]. Initially, about eight oscillations appear on the boundary, this number decreasing to four with the domain size during the experiment. In Fig. 3, only case $\boldsymbol{\Lambda}$, corresponding to Fig. 2(B), shows a contour instability. It corresponds to a typical time scale for disappearance of the order of $7 \mathrm{mi}$ nutes (with an initial radius of $10.5 \mu \mathrm{m}$ ), while case

[Fig. 2(A)] corresponds to 20 minutes for an initial radius of $12 \mu \mathrm{m}$.

Discussion and conclusions. - Collapse of the LO phase does not mean that extraction takes place mostly in this phase, since any extraction implies a lateral flux of chemical equilibration. If cholesterol extraction occurs mostly in the LD phase, the chemical flux is outward, and the collapse occurs without instability. So the experimental evidence of an instability above a threshold value is in favor of a preferred pumping of the LO phase. Typical values of $Q_{i} / R_{0}^{2}$ are $5 \times 10^{-3} \mathrm{~s}^{-1}$ (estimated from the slope at time $t=0$ in Fig. 3) for the case with destabilization ( $(\mathbf{\Delta})$ and $Q_{i} / R_{0}^{2} \sim 6 \times 10^{-4}$ for the case without destabilization (O). The line tension $\gamma$ is about $10^{-5}$ or $10^{-6} \mathrm{~N} / \mathrm{m}$
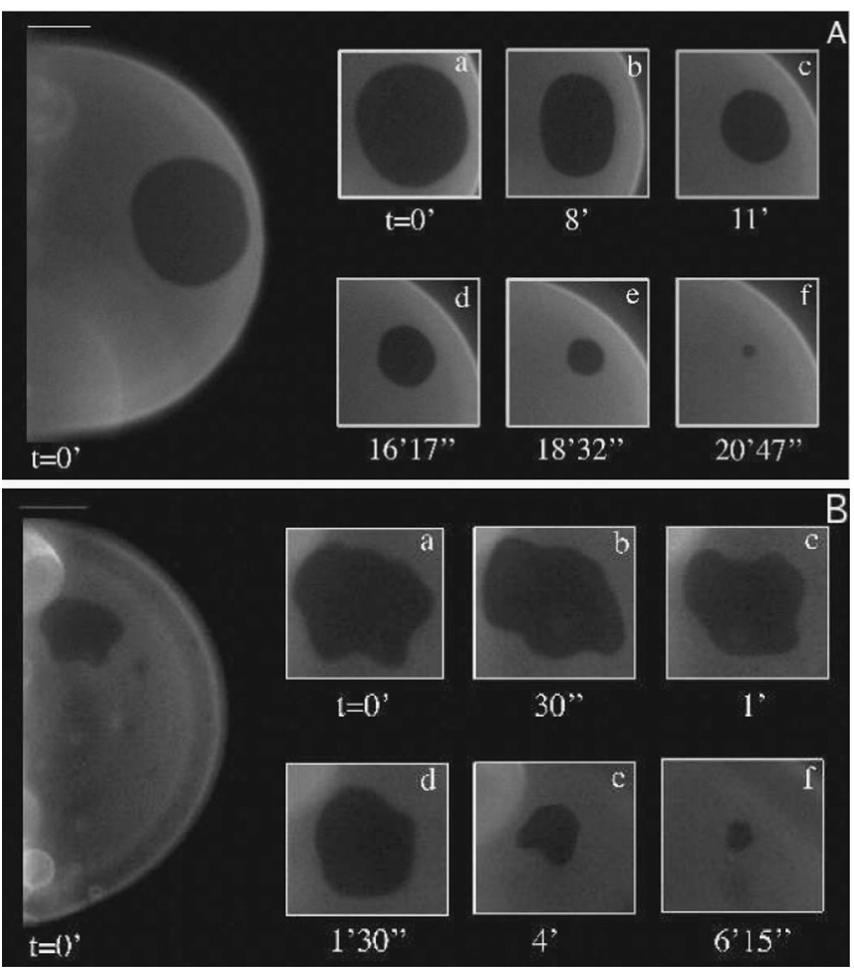

FIG. 2. Evolution of a vesicle containing a LO domain (PC/DiQ/SM/Chol 55:5:20:20, 31C) following a bulk addition of human HDLs. The LO domain appears as a dark round spot. The time counting $(t=0)$ starts about $10 \mathrm{~min}$ after HDL addition to the GUVs (time interval during which domain changes are not yet detected). (A) HDLs bulk concentration $0.1 \mathrm{mg} / \mathrm{ml}$ in apoA1 (adding $100 \mu \mathrm{l}$ of HDLs at $2 \mathrm{mg} / \mathrm{ml}$ in apo-A1): the GUV in fluorescence and (a)-(f) continuous decrease of the LO domain keeping its circular shape (no contour undulations can be resolved). $\quad \mathrm{Bar}=10 \mu \mathrm{m}$. (B) HDLs bulk concentration $0.35 \mathrm{mg} / \mathrm{ml}$ in apo-A1 (adding $350 \mu \mathrm{l}$ of HDLs at $2 \mathrm{mg} / \mathrm{ml}$ in apo-A1): the GUV in fluorescence and (a)-(f) destabilization of the LO domain circular shape upon decreasing - the number of oscillations on the domain boundary being initially about eight decreases with the size of the domain to about four. Bar $=$ $20 \mu \mathrm{m}$.

$[15,16]$. A number of undulations of several units, as observed, implies values of the viscosity of order 10 up to $100 \mathrm{Pas}$, in the range of values suggested for LO domains [26,31]. In the literature, there is a wide dispersion for membrane viscosities which may vary from 0.5 (diffusion of lipids or small proteins [26,31]) to $100 \mathrm{~Pa}$ s (closure of macroscopic pores [32]) depending on the measurement techniques. Moreover, our model predicts an unphysical value of $n_{s} \sim 1$ for the case of slow suction [Fig. 2(A) and - in Fig. 3], which explains why the boundary remains circular during its collapse. Finally, the water drag effect may be given by a heuristic argument: It increases the effective viscosity of each phase as $\mu_{\text {eff }}-\mu_{l} \sim$ $\mu_{w} R_{0} / n h$. It gives here a correction of order $1 \%$, which allows one to neglect the water bath. 


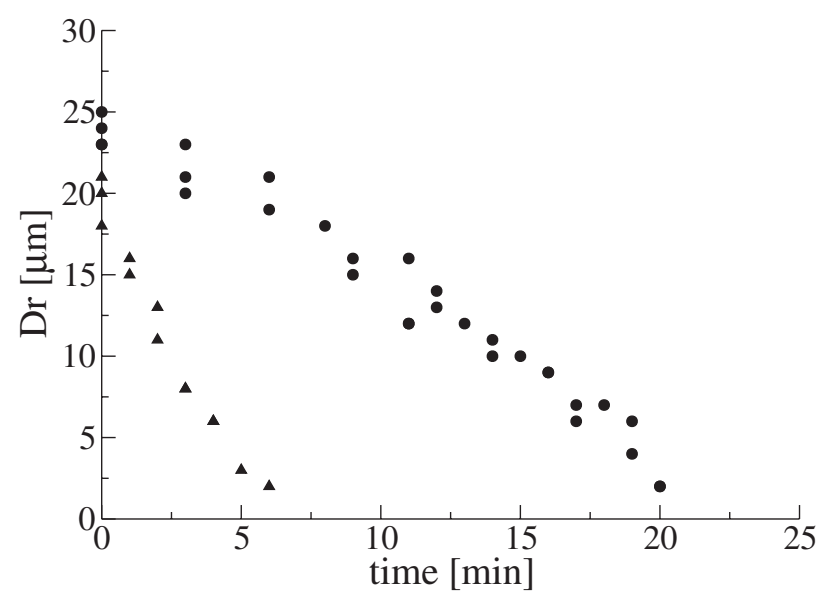

FIG. 3. Kinetics of domain size decrease. Raftlike domain diameter (Dr) as a function of time for different HDL bulk concentrations (in apo-A1): $0.1(\mathbf{O})$ and $0.35 \mathrm{mg} / \mathrm{ml}(\mathbf{\Delta})$.

Despite its theoretical interest, experimental twodimensional Stokes flow has been neglected compared to Hele-Shaw flow. In this Letter, we suggest that some specific processes acting on inhomogeneous bilayers in a situation far from equilibrium can be understood with interfacial hydrodynamical instabilities. The treatment of the 2D Stokes instability simplifies in the case where dissipation in water is neglected, for enough viscous membranes. It turns out to be rather different from the analogous Saffman-Taylor instability [6,7,11]. For our experiment, comparison yields to reasonable viscosity values for the liquid-ordered phase, intermediate between the liquid $[25,26]$ and gel $[26,31]$ estimated values. From a biological point of view, we show that (i) the number of HDLs is critical for the stability, and (ii) the passive cholesterol efflux takes place predominantly in the LO domains. Since there is also some debate about this question, our study is in favor of a preferred cholesterol extraction from LO domains.

*benamar@1ps.ens.fr

[1] G. M. Homsy, Annu. Rev. Fluid Mech. 19, 271 (1987).

[2] Y. Couder, Chaos, Order and Patterns (Plenum, New York, 1991).

[3] E. A. Brener and V. I. Melnikov, Adv. Phys. 40, 53 (1991).

[4] S. Tanveer, J. Fluid Mech. 409, 273 (2000).
[5] J. Casademunt, Chaos 14, 809 (2004).

[6] M. Ben Amar and D. Bonn, Physica (Amsterdam) 209D, 1 (2005).

[7] W.-S. Dai and M. J. Shelley, Phys. Fluids A 5, 2131 (1993); M. J. Shelley, F. R. Tian, and K. Wlodarski, Nonlinearity 10, 1471 (1997); A. Lindner, D. Derks, and M. J. Shelley, Phys. Fluids 17, 072107 (2005).

[8] R. M. Oliveira and J. A. Miranda, Phys. Rev. E 73, 036309 (2006).

[9] J. Bataille, Rev. Inst. Fr. Pét. Ann. Combust. Liq. 23, 1349 (1968).

[10] L. Paterson, J. Fluid Mech. 113, 513 (1981).

[11] P.-G. Saffman and G. Taylor, Proc. R. Soc. A 245, 312 (1958).

[12] D. G. Crowdy and S. Tanveer, J. Nonlinear Sci. 8, 261 (1998).

[13] L. J. Cummings, S. D. Howison, and J. King, Eur. J. Appl. Math. 10, 635 (1999).

[14] H. A. Stone, A. D. Stroock, and A. Ajdari, Annu. Rev. Fluid Mech. 36, 381 (2004).

[15] J. Alexander et al., Phys. Fluids 18, 062103 (2006).

[16] T. Baumgart, S.T. Hess, and W. W. Webb, Nature (London) 425, 821 (2003).

[17] N. Puff et al., Chem. Phys. Lipids 133, 195 (2005).

[18] P.-G. Saffman, J. Fluid Mech. 73, 593 (1976); P.-G. Saffman and M. Delbruck, Proc. Natl. Acad. Sci. U.S.A. 72, 3111 (1975).

[19] H. A. Stone and H. M. McConnell, J. Phys. Chem. 99, 13505 (1995).

[20] D. K. Lubensky and R. E. Goldstein, Phys. Fluids 8, 843 (1996).

[21] G. H. Rothblat et al., J. Lipid Res. 40, 781 (1999).

[22] S. Yokoyama, Biochim. Biophys. Acta 1529, 231 (2000).

[23] C. J. Fielding and P. E. Fielding, Biochim. Biophys. Acta 1533, 175 (2001).

[24] U. Seifert, Adv. Phys. 46, 13 (1997).

[25] W. Helfrich, Z. Naturforsch. C 28, 693 (1973).

[26] R. Lipowsky and E. Sackmann, Structure and Dynamics of Membranes, Handbook of Biological Physics (Elsevier, Amsterdam, 1995).

[27] K. Simons and E. Ikonen, Nature (London) 387, 569 (1997).

[28] P. O’Shea, Phil. Trans. R. Soc. A 363, 575 (2005).

[29] E. Ikonen, Curr. Opin. Cell Biol. 13, 470 (2001).

[30] M.I. Angelova and D.S. Dimitrov, Faraday Discuss. Chem. Soc. 81, 303 (1986).

[31] R. Dimova, B. Pouligny, and C. Dietrich, Biophys. J. 79, 340 (2000).

[32] F. Brochard-Wyart, P.-G. De Gennes, and O. Sandre, Physica (Amsterdam) 278A, 32 (2000). 